\title{
Activated sludge morphology significantly impacts oxygen transfer at the air-liquid boundary
}

\author{
Ken Campbell, ${ }^{1 \star}$ Jianmin Wang, ${ }^{1 \star}$ Guoqiang Liu, ${ }^{2}$ Glen Daigger ${ }^{3 \star}$
}

\begin{abstract}
'Department of Civil, Architectural and Environmental Engineering, Missouri University of Science and Technology, Rolla, Missouri

${ }^{2}$ School of Environment, Jinan University, Guangzhou, Guangdong Province, China

${ }^{3}$ Department of Civil and Environmental Engineering, University of Michigan, Ann Arbor, Michigan
\end{abstract}

Received 26 August 2018; Revised 7 December 2018; Accepted 15 December 2018

Correspondence to: Jianmin Wang, Department of Civil, Architectural and Environmental Engineering, Missouri University of Science and Technology, Rolla, MO.

Email: wangjia@mst.edu

K. Campbell received Chancellor's Fellowship to cover his tuition during the course of this research.

*WEF Member

Published online 14 February 2019 in Wiley Online Library (wileyonlinelibrary.com)

DOI: 10.1002/wer.1066

(c) 2019 Water Environment Federation

\section{- Abstract}

Oxygen transfer is a key process determining the energy use of a biological wastewater treatment process. In this research, we investigated the effect of sludge morphology, especially the role of filamentous microorganisms, on oxygen transfer using benchscale complete-mix activated sludge reactors with solids retention times (SRTs) of 10-, 20 -, and 40-days, respectively. Results indicated 5\%-10\% reduced aeration need in the 40-day SRT reactor, compared with 10- and 20-day SRT reactors to maintain the same dissolved oxygen level, due to the improvement in sludge settleability and oxygen transfer efficiency (OTE). Filamentous microorganisms adversely impacted OTE via an increase in apparent viscosity of the mixed liquor, which resulted in an increase in the air bubble size and liquid film thickness and, therefore, limited oxygen transfer at the air-liquid boundary. A statistical analysis also confirmed that the mixed liquor viscosity is a statistically significant parameter links to OTE. () 2019 Water Environment Federation

\section{- Practitioner points}

- Filamentous organisms reduce oxygen transfer via increasing mixed liquor viscosity, which increases air bubble size and liquid film thickness at the air-liquid boundary.

- Increasing solids retention time reduces filament density.

- As a result, very long solids retention time promotes oxygen transfer.

\section{- Key words}

activated sludge; filamentous microorganisms; oxygen transfer efficiency; viscosity

\section{INTRODUCTION}

IN our previous research, we reported that long-term low DO inhibited the decay of both ammonia oxidation bacteria $(\mathrm{AOB})$ and nitrite oxidation bacteria (NOB), resulting in greater nitrification capacity (Liu \& Wang, 2013, 2015). Long-term low DO aeration also shifted the NOB community to species that have a greater oxygen affinity (Liu \& Wang, 2013). As a result, low DO did not significantly impact overall nitrification performance, provided the microbial community had adequate time to adapt to the selective environmental pressures. Through the implementation of the low DO aeration strategy, the DO deficit between the gas and liquid phase is increased, enhancing the mass transfer of oxygen into the mixed liquor. Theoretically, improvements in oxygen transfer efficiency (OTE) should be proportional to the increase in the DO deficit. However, our experimental observations revealed an increase in OTE less than anticipated (Liu, Wang, \& Campbell, 2018). Careful review of low DO aeration results suggested that the growth of filamentous microorganisms negatively impacted oxygen transfer performance (Liu et al., 2018). It was initially anticipated that filamentous microorganisms would improve the OTE due to their greater surface area-to-volume ratio, which might reduce oxygen diffusional resistances (Martins, Pagilla, Heijnen, \& van Loosdrecht, 2004). However, our observations suggested the opposite direction. Clearly, these results suggested that microbial morphology played an important role in oxygen mass transfer in the activated sludge process. 
Several hypotheses arose from our previous findings. First, it was anticipated that the settled sludge volume fraction after 30 min of settling time, $\mathrm{SV}_{30}$, and by extension the sludge volume index, SVI, would be related to the OTE, with increasing values of these parameters resulting in reductions in the OTE. The link between the $\mathrm{SV}_{30}$, SVI, and filamentous organism densities has been clearly established (Palm, Jenkins, \& Parker, 1980; Sezgin, 1982; Sezgin, Jenkins, \& Parker, 1978). However, associations between the $\mathrm{SV}_{30}$, SVI, and OTE have not been elucidated. Similarly, based on anecdotal evidence from our initial study, it was anticipated that the mixed liquor viscosity might be related to differences in OTE observed. Activated sludge mixed liquor is inherently a suspension. It has been shown that as the volume fraction of particles in suspension increase, the viscosity increases (Hiemenz \& Rajagopalan, 1997; Macosko, 1994). This increase in viscosity has been demonstrated to reduce volumetric mass transfer coefficients in conventional activated sludge and membrane bioreactor processes (Krampe \& Krauth, 2003; Wagner, Cornel, \& Krause, 2002). While we speculate that filamentous organisms might have a deleterious impact on the viscosity and subsequently the OTE, the detailed link between them has not been investigated.

The OTE is vitally important because it dictates energy use and cost of the activated sludge process. In 2011, 30.2 TW-h of electricity was consumed to treat municipal wastewater by centralized treatment plants in the United States (US Department of Energy, 2014). For a conventional secondary activated sludge treatment process, aeration contributes approximately 50\% of the total plant energy consumption (WEF and ASCE, 2010). Substantial energy savings can be realized if the air delivery requirements can be reduced by increasing OTE. In an age when the issue of climate change is in the forefront, the reduction in greenhouse gas emissions afforded by increases in the OTE can be significant.

The primary goal of this study was to track the morphological differences between complete-mix activated sludge reactors operated at different SRTs to ascertain their impacts on OTE, with specific attention given to sludge settleability expressed as a sludge volume fraction from a 30 -min settling test $\left(\mathrm{SV}_{30}, \%\right)$ and the mixed liquor apparent viscosity. This work fills an important gap, because the body of work relating the settleability to OTE is scant. It is anticipated that the $\mathrm{SV}_{30}$ is a reasonable corollary to the hydrodynamic radius of the suspended microbial aggregate, directly influencing the OTE. This study is intended to improve understanding of the role of sludge morphology on the mass transfer of oxygen from the gas phase to the microbial floc. A second goal is to provide practical tools which can be used by process operators to assess the energy use for their process.

\section{MATERIALS AND METHODS}

\section{Reactor setup}

Three $31.5 \mathrm{~L}$ complete-mix, baffled reactors with individual solids retention times (SRTs) of 10, 20, and 40-days were used for this research. The specific SRT of each reactor was maintained by wasting a fixed volume of the mixed liquor from each reactor daily. These reactors were seeded with activated sludge from the Southeast Wastewater Treatment Plant, located in Rolla, Missouri, USA, which receives predominantly domestic wastewater. Mixing within each reactor was performed with a six-bladed Rushton turbine driven by variable speed, 1/25-Hp permanent magnet 90 VDC gear motors (Baldor GP232001). The mixer rotational speed was adjusted to achieve a target Root Mean Square (RMS) velocity gradients, $\bar{G}$, of $150 \mathrm{~s}^{-1}$ in tap water. This mixing intensity was chosen as it was found to be reflective of typical mixing intensities of full-scale treatment facilities (Grady, Leslie, Daigger, \& Lim, 1999). Actual mixing intensities likely varied with fluctuations in mixed liquor apparent viscosity. Measurement of the mixer rotational speed was performed periodically with a hand-held digital photo tachometer (DT2234C). Air was introduced into each reactor by a 0.61-m (2-ft) long Pentair Aquatic Ecosystems Bio-Weave diffuser hose installed directly below the mixer. Air flow into each reactor was measured with a 5.0 SCFH variable area rotameter (Cole Parmer) and adjusted multiple times daily, to maintain a dissolved oxygen (DO) concentration of $2 \mathrm{mg} / \mathrm{L}$. Routine reactor maintenance was performed, which included daily brushing of reactor surfaces and weekly cleaning of chemical feed apparatus as detailed by Gabb, Ekama, Jenkins, \& Marais (1989), to limit the formation of biofilms which might adversely impact the microbial kinetics of the system.

A 378 L HDPE tank was used to prepare a common synthetic wastewater for all reactors, and a $75 \mathrm{~W}$, submersible, thermostatically controlled heating element was utilized to maintain the bulk liquid temperature at $20 \pm 1^{\circ} \mathrm{C}$. The synthetic wastewater was continuously fed into each reactor at a flow rate of $63 \mathrm{~L} /$ day via a variable speed peristaltic pump having a common pump head (Cole Parmer Masterflex Model 7553-70 with 16 mm Masterflex Norprene tubing element) to achieve a hydraulic retention time (HRT) of $12 \mathrm{hr}$ for each reactor. Glucose (organic carbon source) and ammonium bicarbonate (nitrogen source) were applied at concentrations of $180.4 \pm 9.2 \mathrm{mg} \mathrm{COD} \mathrm{L}^{-1}$ and $42.4 \pm 1.71 \mathrm{mg} \mathrm{N} \mathrm{L}^{-1}$, respectively. Trace metals and buffers were added as follows: $\mathrm{K}_{2} \mathrm{PO}_{4}, 4.0 \mathrm{mg} \mathrm{PO}{ }^{3-} \mathrm{L}^{-1} ; \mathrm{MnCl}_{2} \bullet 4 \mathrm{H}_{2} \mathrm{O}$, $0.2 \mathrm{mg} \mathrm{Mn} \mathrm{L}^{-1} ; \mathrm{MoCl}_{5}, 0.04 \mathrm{mg} \mathrm{Mo} \mathrm{L}{ }^{-1} ; \mathrm{CoCl}_{2}, 0.001 \mathrm{mg} \mathrm{Co} \mathrm{L}{ }^{-1}$; $\mathrm{ZnCl}_{2}, 0.05 \mathrm{mg} \mathrm{Zn} \mathrm{L}^{-1} ; \mathrm{FeSO}_{4} \cdot 7 \mathrm{H}_{2} \mathrm{O}, 0.005 \mathrm{mg} \mathrm{Fe} \mathrm{L}{ }^{-1}$. Calcium and magnesium were present in sufficient quantities within the tap water utilized for production of the synthetic wastewater. Sodium carbonate was utilized as necessary to supplement the buffer capacity of the feed solution and maintain optimum nitrification rates. The $\mathrm{pH}$ of the reactor mixed liquor was maintained approximately 7.0.

Influent, effluent, and mixed liquor testing was performed throughout the duration of the experiment. Effluent testing was performed routinely utilizing Hach TNT Plus vial testing system with a Hach DR 2800 spectrophotometer: Hach TNT 822 for COD; Hach TNT 830 for ammonia-nitrogen concentrations; Hach TNT 835 for nitrate-nitrogen; and Hach TNT 839 for nitrite-nitrogen. Reactor temperature and operational DO concentrations were monitored numerous times daily utilizing a polarographic DO probe (YSI model 58 with model 5239 probe). The rotameter and DO probe response time were periodically evaluated to assure conformance to the ASCE/EWRI standards. Mixed liquor and effluent suspended solids testing was performed 
in conformance with SM 2540 D (APHA et al. 2000). Mixed liquor settleability and sludge volume index (SVI) were determined in accordance with SM 2710 B and C, respectively (APHA, 2000).

\section{Oxygen demand and transfer efficiency determination}

Process oxygen demand and OTE were determined as previously described (Liu et al., 2018). In brief, oxygen consumption within the reactor was related to the degradation of organic carbon (glucose), nitrification (ammonium bicarbonate), and biomass production. Oxygen demand for glucose degradation was calculated based in Equation 1:

$$
R_{\mathrm{COD}}=Q\left(S_{\mathrm{COD}}^{\mathrm{o}}-S_{\mathrm{COD}}\right) \text {, }
$$

where $R_{\mathrm{COD}}$ is the oxygen demand associated with carbon substrate oxidation (mg/day); $Q$ is the volumetric flow rate (LPD); $S_{\mathrm{COD}}^{o}$ is the influent COD concentration $(\mathrm{mg} / \mathrm{L}) ; S_{\mathrm{COD}}$ is the effluent COD concentration $(\mathrm{mg} / \mathrm{L})$.

The oxygen demand associated with nitrification was determined using Equation 2.

$$
R_{\mathrm{N}}=4.57 Q S_{\mathrm{NO}_{3}^{-}}+3.43 Q S_{\mathrm{NO}_{2}^{-}} .
$$

where $R_{\mathrm{N}}$ is the oxygen demand associated with nitrogen oxidation; $S_{\mathrm{NO} 3-}$ is the effluent nitrate concentration $\left(\mathrm{mg} \mathrm{NO}_{3}{ }^{-} \mathrm{L}^{-1}\right)$; and $\mathrm{S}_{\mathrm{NO} 2}$ is the effluent nitrite concentration $\left(\mathrm{mg} \mathrm{NO}_{2}{ }^{-} \mathrm{L}^{-1}\right)$.

The oxygen demand will be reduced as a consequence of biomass production $\mathrm{P}_{\mathrm{x}, \mathrm{bio}}$ within the reactor. The $P_{\mathrm{x}, \text { bio }}$ was calculated based on observed biomass yield by measurement. The theoretical oxygen demand of the biomass was taken to be $1.42 \mathrm{~g} \mathrm{O}_{2} \mathrm{~g}^{-1}$ biomass.

$$
R_{\text {bio }}=-1.42 P_{\mathrm{x}, \mathrm{bio}},
$$

where $R_{\mathrm{bio}}$ is the oxygen credit associated with the net biomass production ( $\mathrm{mg} /$ day).

The overall oxygen demand was calculated using Equation 4.

$$
R_{\mathrm{O}_{2}}=R_{\mathrm{COD}}+R_{\mathrm{N}}+R_{\text {bio }} .
$$

The OTE was determined as the ratio of oxygen sinks to the mass flow of oxygen supplied to the reactor using Equation 5.

$$
\mathrm{OTE}=\frac{Q\left(C-C^{\circ}\right)+R_{\mathrm{O}_{2}}}{M_{\mathrm{O}_{2}}},
$$

where $C$ is the dissolved oxygen in the effluent (mg/day); $C^{0}$ is the dissolved oxygen in the feed (mg/day); and $M_{\mathrm{O} 2}$ is the oxygen mass flow delivered to the system via aeration (mg/day).

The aforementioned protocol for the determination of OTE was utilized in lieu of guidelines presented in the ASCE/ EWRI 18-96 document (ASCE, 1997). The ASCE/EWRI document provides some guidance regarding OTE determination, but it is recognized that results from the testing protocols can be variable based on process conditions. The methodology presented herein has the benefit of allowing the assessment of the OTE based on long-term process performance, whereas the ASCE/EWRI methods only give snapshots. Furthermore, the presented methodology was found to be more easily adaptable to small geometry reactors utilized within the scope of this work.

\section{Volumetric mass transfer coefficient determination}

Testing was performed to assess the oxygen transfer characteristics of the reactors under different operating conditions. The ASCE/EWRI methodology for the determination of oxygen transfer in clean water was utilized for this assessment (ASCE, 2007). All three operational reactors were tested with tap water prior to the commencement of operation. A reactor having the same geometry, diffuser configuration, mixer, etc., as the three operational reactors was utilized for testing the effluent from the three operational reactors. Each reactor was filled with the working fluid (tap water or reactor effluent) and mixed at an intensity of $\bar{G}=150 \mathrm{~s}^{-1}$. The airflow rate was maintained at a constant, preselected rate for the duration of the test. The dissolved oxygen concentration was monitored as a function of time. Replicate tests were performed for all experiments.

\section{Viscosity measurement}

The apparent viscosity of the mixed liquor was determined utilizing an Anton Paar MCR 302, configured for use with parallel plates. Each sandblasted plate was circular, having a diameter of $49.987 \mathrm{~mm}$. The bottom plate was fixed, and the top plate was allowed to rotate. The gap between the upper and lower plates was set to 1.5 times the maximum particle size to provide repeatable results and limit the formation of secondary flows. The maximum particle size was determined via brightfield microscopy and image analysis of a statistically significant sample of floc images. The temperature of the plates and sample was maintained at $20.0^{\circ} \mathrm{C}$ by a thermostatically controlled circulating water bath. The rotation of the upper plate was controlled based on the applied shear rate. Initially, a shear rate of $150 \mathrm{~s}^{-1}$ was applied to the sample for a duration of $15 \mathrm{~s}$. The shear rate was then linearly decreased from 150 to $1.0 \mathrm{~s}^{-1}$. The resulting torque was recorded. Based on plate geometry, applied shear rate and measured torque, the shear stress and apparent viscosity were calculated. Apparent viscosity data were reported at a shear rate of $150 \mathrm{~s}^{-1}$, which is reflective of the target mixing intensity utilized in the reactors.

\section{RESULTS AND DISCUSSION}

\section{Reactor operations}

The starting mixed liquor suspended solids (MLSS) concentration for each reactor was high $(>4,000 \mathrm{mg} / \mathrm{L})$ and was allowed to reach equilibrium with routine wasting. Figure 1 shows that for the 10- and 20-days SRT reactors, the MLSS concentrations stabilized between Day 100 and 150 at $741 \pm 67 \mathrm{mg} / \mathrm{L}$ and $1,387 \pm 121 \mathrm{mg} / \mathrm{L}$, respectively. The 40 -days SRT reactor MLSS stabilized after approximately Day 300 at $2,578 \pm 155 \mathrm{mg} / \mathrm{L}$. SVIs for the 10- and 20-days reactors generally increased from Day 0 to 300 , stabilizing at $1,343 \pm 1,278$ and $674 \pm 123 \mathrm{~L} / \mathrm{g}$, respectively. The 40-days reactor saw a general increase in SVI to approximately $355 \mathrm{~L} / \mathrm{g}$ at Day 300 . The observed airflow rates generally followed the patterns seen with the MLSS concentrations, decreasing (10-d and 20-days SRT) or remaining relatively constant $(\mathrm{SRT}=40$-days) until Day 300 . 

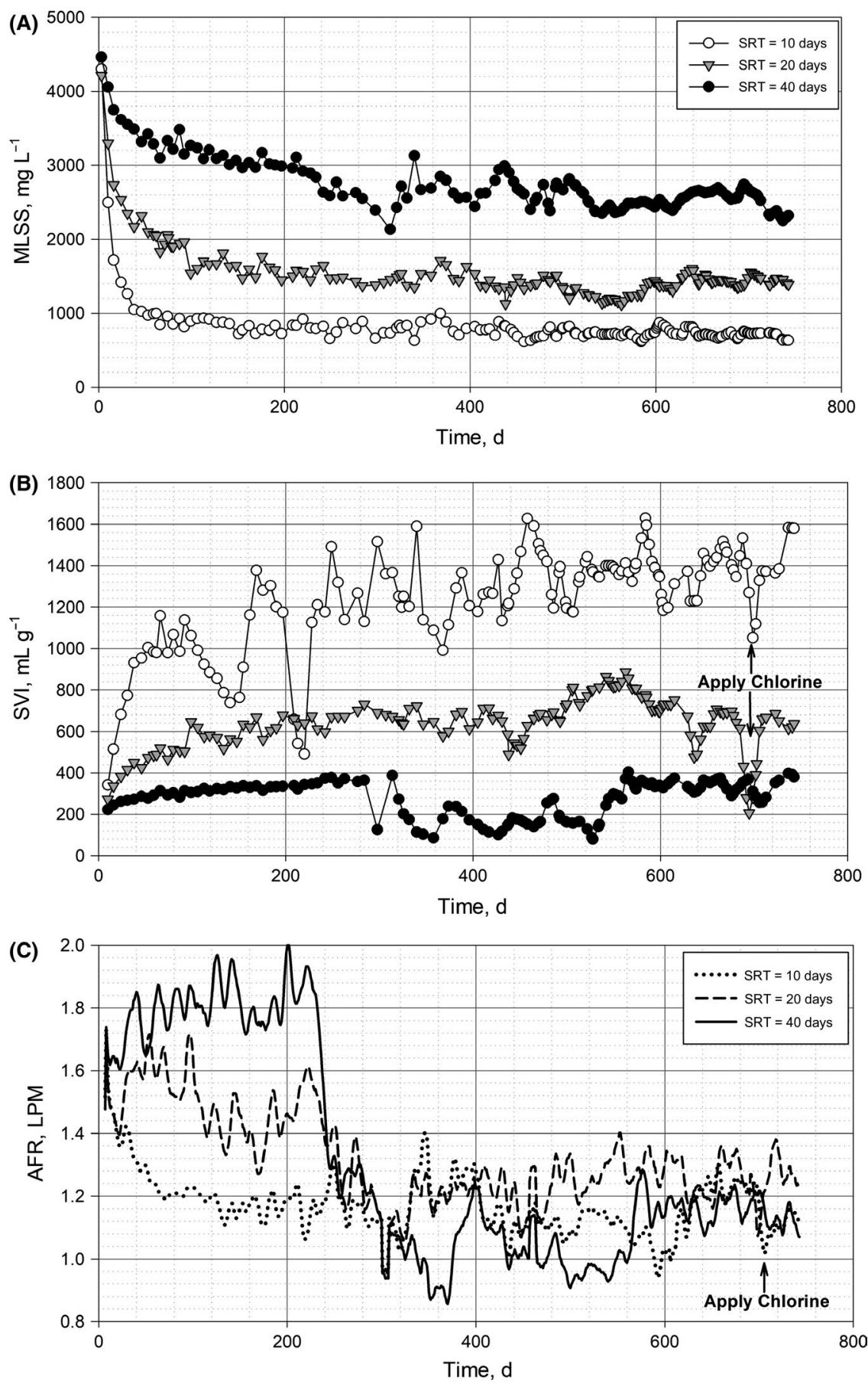

Figure 1. (a) Mixed Liquor Suspended Solids (MLSS), (b) Sludge Volume Index (SVI) and (c) Airflow Rate (AFR), with respect to time for: Reactor $1, \mathrm{SRT}=10$ days; Reactor 2, SRT $=20$ days; and Reactor $3, \mathrm{SRT}=40$ days.

At Day 300, statistically significant reductions in airflow rates for the 20 and 40 days reactors were observed. Prior to this, the 20 and 40 days reactors had airflow rates of $1.45 \pm 0.16 \mathrm{Lpm}$ (liter per minute) and $1.82 \pm 0.25 \mathrm{Lpm}$, respectively. After Day 300, these reactors exhibited airflows rates of $1.23 \pm 0.10$ and $1.06 \pm 0.11 \mathrm{Lpm}$, respectively. These reductions corresponded to a $14 \%$ reduction in the 20 -days SRT reactor and a $36 \%$ reduction in the 40 days SRT reactor.
During and after this period, the 10-d SRT reactor exhibited no significant change in airflow rate, averaging $1.15 \pm 0.10$ Lpm. A $t$ test with significance level, $\alpha=0.05$, was conducted to compare airflow rates pre- and post-adjustment demonstrating no significant change for the 10-days reactor $[t(423)=1.81, p=0.07]$, but significant changes for the 20-d $\left[t(423)=18.47, p=2.81 \times 10^{-56}\right)$ and 40 -days $[t(139)=64.54$, $\left.p=1.6 \times 10^{-105}\right]$ reactors. The changes in the airflow rate were 
accompanied by increased variability in the SVIs, especially for the 40 days SRT reactor.

These decreases were significant, resulting in an airflow in 20 -days SRT reactor that was only $8 \%$ higher than 10 -days SRT reactor. Furthermore, the airflow rate for 40-days SRT reactor was $7 \%$ less than the airflow for 10-days SRT reactor. This is significant because the oxygen demand for each reactor should increase with the increasing SRT. A theoretical assessment of oxygen demand reveals that a 20-day and 40-day SRT should require $9 \%$ and $16 \%$ more oxygen than for the 10 -day SRT. This theoretical difference is driven by the more predominant role of endogenous respiration at long SRTs. While the relative air supply rate for the 20-days SRT reactor matched the theoretical calculations, the actual air supply rate for the 40-days reactor was $20 \%$ less than what was anticipated based on theoretical calculations, accounting for substrate utilization, biomass production, endogenous respiration, etc.

The reactor geometries were identical and did not change for the duration of the testing. To rule out diffuser fouling or variation as a potential cause for the observed differences in the air delivery rate, the diffusers for 10-day and 40-day reactors were swapped on Day 377. The diffusers for 10-day and 40day reactors were then switched back to their original positions at Day 630. No significant deviations in air supply rates were observed with the subsequent swap of diffusers, ruling out diffuser fouling or variation as the cause for the observed system response.

The operational parameters (F/M, SRT, HRT, temperature, etc.) for all reactors were held constant for the duration of testing. The observed adjustments are therefore not directly related to these parameters and represent a deviation from expected theoretical behavior. All other things being equal, it is apparent that differences in fundamental characteristics associated with the mixed liquor were responsible for observed system response. Seasonal and temporal variations in the microbial community makeup have been observed in large scale wastewater treatment facilities, characterized by shifts in operational taxonomic groups as a result of competition, ecological nichegroup interactions, etc. (Ju \& Zhang, 2014; Valentín-Vargas, Toro-Labrador, \& Massol-Deyá, 2012). It is hypothesized that the observed adjustments and the subsequent cyclic variations in reactor airflow, SVI, etc. were directly related to microbial culture dynamics.

At Day 690, chlorine (as $\mathrm{NaOCl}$ ) was added to the 20-days SRT reactor. Between Day 697 and 699, chlorine (as $\mathrm{NaOCl}$ ) was also added to 10-days SRT reactor. This was done to validate the role of filamentous microorganisms on oxygen transfer performance. Chlorine was dosed at a rate of $0.006 \mathrm{~g} \mathrm{Cl}_{2} \mathrm{~g}^{-1}$ MLSS in both reactors, resulting in a subsequent decrease in the air flowrate and the SVI (see Figure 1). During and immediately after the chlorine dose, the performance of the reactor, including the nitrification capacity of the sludge, remained unimpacted. The observed system response to chlorine addition clearly demonstrates the significant impact of the filamentous microorganisms on the OTE. Marginal changes in the MLSS concentrations were observed. The immediate reduction in the SVI was a direct result of the reduction in filament density.
The $27 \%$ and $24 \%$ reduction in the airflow delivery rates for the 10-days and 20-days SRT reactors, respectively, was associated with an increase in the mixed liquor OTE, which was associated with the reduction in filament densities.

\section{Sludge settleability and the OTE}

The volume of sludge after 30 min settling time, $\mathrm{SV}_{30}(\%)$, was compared with various operational parameters. Most significantly, the $\mathrm{SV}_{30}$ was linearly related to the OTE. As detailed in Figure 2, when $\mathrm{SV}_{30}$ increased from 20 to 100, the OTE decreased from $5.04 \%$ to $3.65 \%$, which is a $28 \%$ reduction. Looking closely at the figure, the $\mathrm{SV}_{30}$ generally increased with decreasing SRT. The 10-days and 20-days reactors generally had a high $\mathrm{SV}_{30}$ and low OTE, while the 40-days reactor had a lower average $\mathrm{SV}_{30}$ but higher OTE. These parameters were prone to fluctuations, however, which were a direct result of cyclical blooms of filamentous microorganisms.

The $\mathrm{SV}_{30}$ is a good indicator of the abundance of poorly settling microorganisms within the sludge. Filamentous microorganisms, which have a high surface area-to-volume ratio, are notorious for impeding sludge settling. Any turbulent motion of the bulk liquid will resuspend filamentous organisms, especially at higher filament densities. Therefore, a high $\mathrm{SV}_{30}$ is a reasonable indication of the filamentous organism density, provided that viscous bulking is not present. This observation is significant because the presence and concentration of filaments within the sludge also directly correlates with poor OTE in the reactors.

The relationship between filamentous microorganisms and OTE can be further supported by a qualitative evaluation of activated sludge morphology. Examining Figure 3, it can be observed that low OTE and high SVI coincided with high filamentous microorganism densities. For example, Day 525

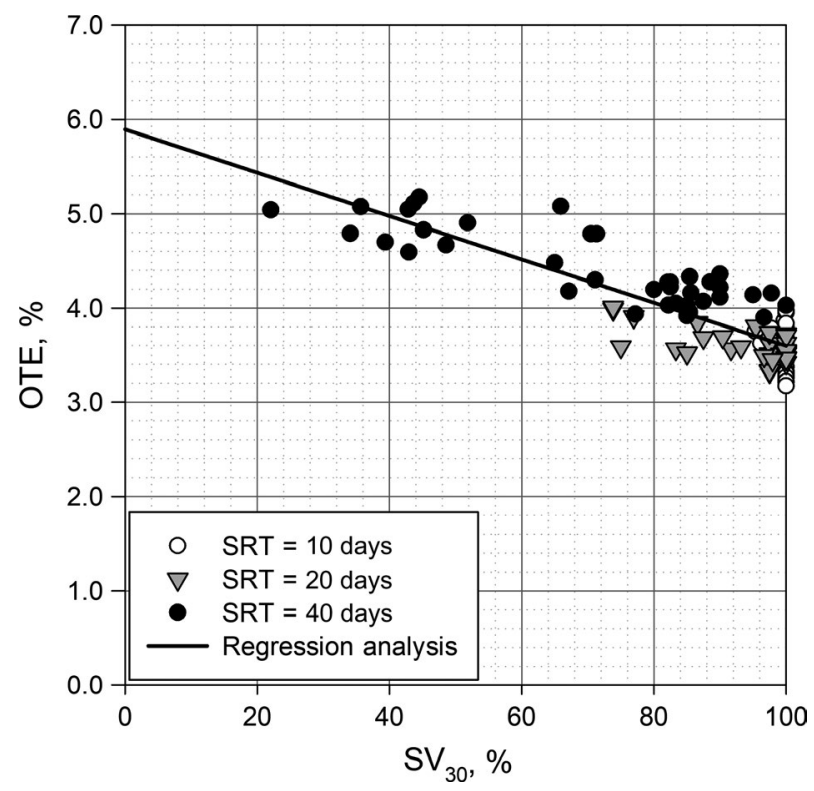

Figure 2. Sludge volume after 30 min settling expressed as a percentage $\left(\mathrm{SV}_{30}, \%\right)$ versus the OTE, with linear regression analysis $\left(R^{2}=0.74\right)$. Data reported since the commencement of viscosity testing (Day 400). 


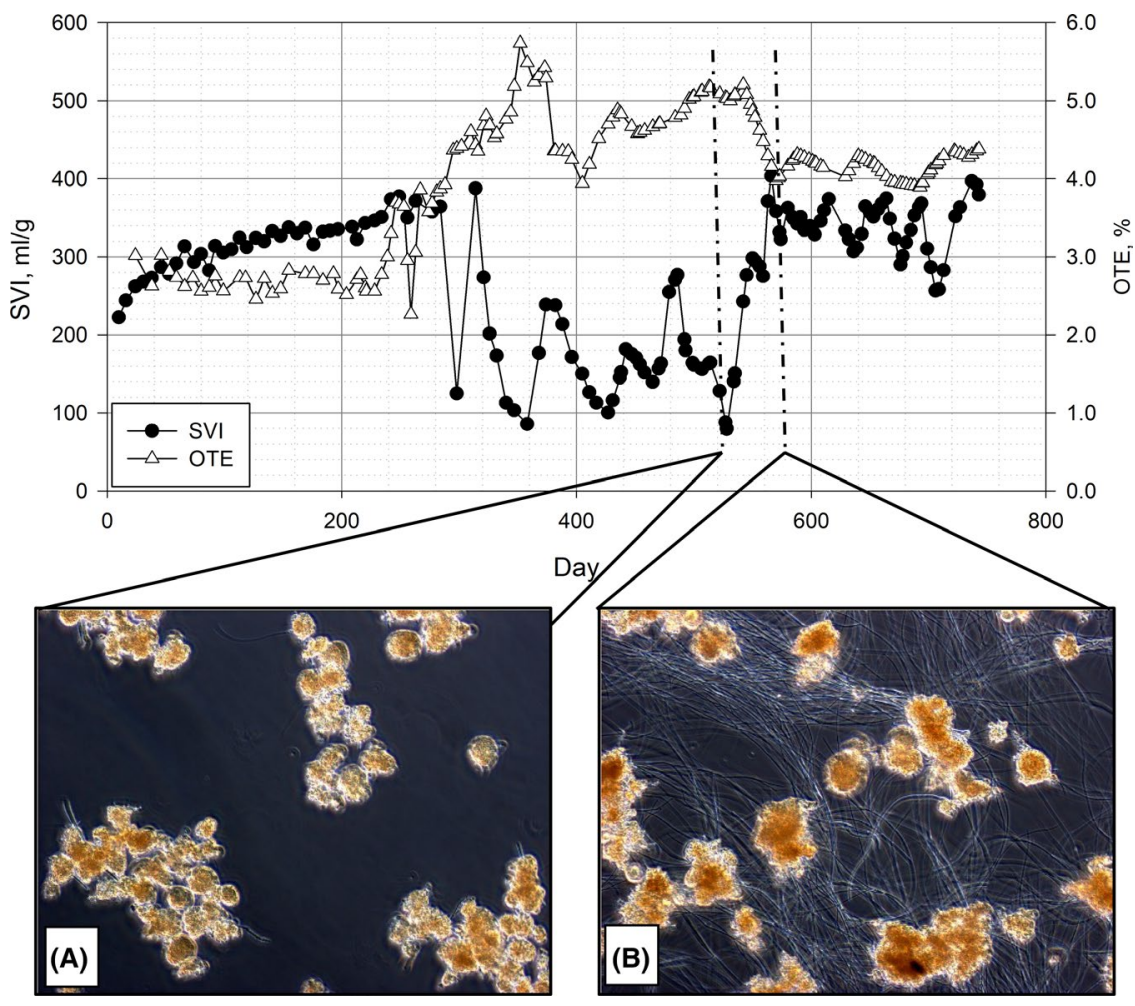

Figure 3. Microscopic investigation of floc morphology at different times during reactor operation for the 40-day reactor: (a) Day 525; (b) Day 563. Fluctuations in both the OTE and SVI correlate well with the presence or absence of filamentous organisms.

imagery reveals that no filamentous organisms were present, corresponding with a high OTE and low SVI. At Day 563, the filament density was high, resulting in low OTE and high SVI. This same trend continued as the 40-days SRT reactor cycled through periods of high filament density and the disappearance of the filaments. It must be noted that, for other reactors, filament density ebbed and flowed; however, they never fully disappeared as was observed for 40-days SRT reactor. As a consequence, the 40-days SRT reactor data span the full range of possible values, whereas 10 -days and 20-days SRT reactors had data confined to a narrow range of observed values.

Filamentous microorganisms have been proven problematic for operations because of poor settling (Palm et al., 1980; Sezgin et al., 1978). Filamentous microorganisms have been shown to have increased EPS production which negatively impacts submerged membrane performance (Meng et al., 2006). It has been suggested that filamentous microorganisms may play a role in the transfer of oxygen in MBRs; however, this assertion was not experimentally verified (Sarioglu, Insel, Artan, \& Orhon, 2009). We previously observed that the filamentous microorganisms induced from low DO aeration reduced OTE (Liu et al., 2018). SV 30 measurements, coupled with the qualitative observation of filament density via microscopic analysis for the reactor operated under a regular DO level of $2 \mathrm{mg} / \mathrm{L}$, confirmed filamentous microorganisms as the cause of impeded OTE for both low and high DO operations.

\section{Apparent viscosity}

$\mathrm{SV}_{30}$ was also found to loosely correlate with the mixed liquor apparent viscosity, $\mu_{\text {app}}$. As seen in Figure $4 \mathrm{a}, \mu_{\text {app }}$ of the mixed liquor generally increased with increasing $\mathrm{SV}_{30}$. Data for the 40d SRT reactor span the entire domain of possible settleability. This is primarily due to the cycling of filament densities throughout the duration of the experiment. For 10 days SRT and 20 days SRT reactors, the cycling was not as pronounced and filament densities were generally high, resulting in the grouping of data near the extrema of the plot.

The question that subsequently arises is what specific quality of filamentous microorganisms results in decreased OTE. Looking further at the $\mathrm{SV}_{30}$ results in comparison with apparent viscosity data in Figure $4 \mathrm{a}$, it is evident that as the $\mathrm{SV}_{30}$ increases, the apparent viscosity increased. The observed system response generally follows an Einstein's Equation (Equation 6) for the viscosity of dispersions, with the $\mathrm{SV}_{30}$ measurement as a corollary to the volume fraction of the dispersion. For mixed liquor, the floc-filament aggregates are significantly larger than the solute molecules. Based on the observed experimental results, the solute viscosity, $\mu_{\mathrm{o}}$, was $0.00102 \mathrm{~Pa} \mathrm{~s}$, yielding a least squares regression fit for $k=2.2\left(R^{2}=0.20\right)$.

$$
\frac{\mu_{\text {app }}}{\mu_{\mathrm{o}}}=1+k \phi,
$$

where $\phi$ is the volume concentration of the particles. 

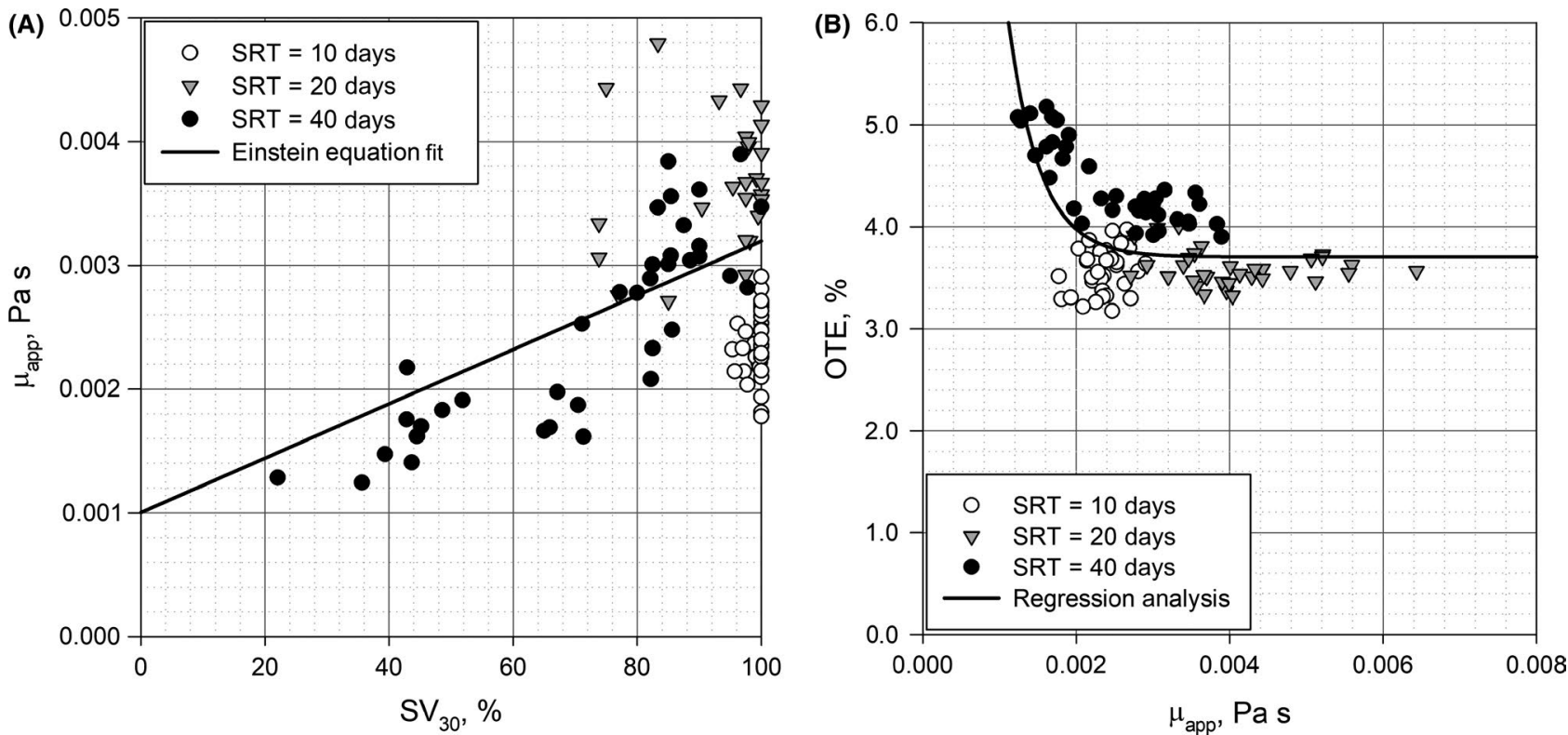

Figure 4. (a) Sludge volume after 30 min settling expressed as a percentage (SV30,\%) versus viscosity, with line described by the Einstein Equation for Viscosity of Dilute Suspension ( $\left.\mu_{\mathrm{o}}=0.001002 \mathrm{~Pa} \mathrm{~s}, k=2.2 ; \varphi=\mathrm{SV}_{30} / 100\right)$ with $R^{2}=0.20$; (b) Apparent Viscosity ( $\left.\mu_{\mathrm{app}}\right)$ versus the OTE. An exponential decay in the OTE was observed with increasing viscosity $\left(R^{2}=0.48\right)$. As the viscosity increased from 0.00136 to $0.00393 \mathrm{~Pa}$ s, a $30 \%$ decrease in the OTE was observed. Data reported since the commencement of viscosity testing (Day 400$)$.

There is dispersion in the $\mathrm{SV}_{30}$ data as it related to the apparent viscosity. Several factors likely play a role in causing this dispersion. First, the floc-filament aggregates are not rigid spheres as initially proposed by Einstein. When the floc-filament aggregates are acted upon by hydrodynamic forces or particle-to-particle interactions occur, the aggregates can deform or disperse, resulting in a dissipation of energy (Hiemenz \& Rajagopalan, 1997; Macosko, 1994). Furthermore, filamentous microorganisms are inherently non-spherical, with large aspect ratios, influencing the apparent viscosity based on particle orientation in the flow field (Macosko, 1994). Finally, the $\mathrm{SV}_{30}$ was measured after $30 \mathrm{~min}$ of settling and does not represent the practical particle volume fraction. Many of the data points for 10-days and 20-days reactors are located at the threshold $\mathrm{SV}_{30}$ of 100 , indicating no settling of the sample. The use of longer settling times becomes problematic due to the risk of denitrification which hinders settling and adversely impacts the outcome of the test. The use of a diluted settleability test might provide a better correlation with the hydrodynamic radius; however, this testing may not truly reflect the in-situ condition in the realistic bioreactor.

The apparent viscosity, $\mu_{\text {app }}$, played a significant role in determining the OTE of the system, as illustrated in Figure $4 \mathrm{~b}$. An exponential decay function was found to reflect the trend of the system response $\left(R^{2}=0.48\right)$, with a practical limit in the system OTE of $6.1 \%$ at a $\mu_{\text {app }}=0.00102 \mathrm{~Pa}$ s (water solution without any particles). With the increase in apparent viscosity from 0.00136 to $0.00393 \mathrm{~Pa}$ s, OTE decreased by $30 \%$. As discussed above, this increase in apparent viscosity is related to the density of filamentous microorganisms in the mixed liquor. This finding is significant because deleterious impacts to the
OTE as a result of increased apparent viscosity are typically not considered to be significant at MLSS concentrations utilized within the scope of this study (Krampe \& Krauth, 2003).

The apparent viscosity can affect the mass transfer of oxygen within the mixed liquor in a number of ways. First, it can impact bubble formation in Newtonian and non-Newtonian fluids, resulting in larger diameter bubbles. Generally, the bubble diameter is directly proportional to the viscosity of the dispersion (Bhavaraju \& Russel, 1978).

$$
d_{\mathrm{b}} \propto\left[\frac{\sigma^{0.6}}{\left(\frac{P}{V_{\mathrm{R}}}\right)^{0.4} \rho_{\mathrm{L}}^{0.2}}\right]\left[\frac{\mu_{\mathrm{app}}}{\mu_{\mathrm{g}}}\right]^{0.1},
$$

where $d_{\mathrm{b}}$ is the bubble diameter $(\mathrm{m}) ; \sigma$ is the liquid surface tension $(\mathrm{N} / \mathrm{m}) ; P$ is the energy input into the system $(\mathrm{W}) ; V_{\mathrm{R}}$ is the reactor volume $\left(\mathrm{m}^{3}\right) ; \rho_{\mathrm{L}}$ is the liquid density $\left(\mathrm{kg} / \mathrm{m}^{3}\right) ; \mu_{\text {app }}$ is the apparent viscosity of the liquid phase and/or dispersion (Pa s); $\mu_{\mathrm{g}}$ is the gas viscosity (Pa s).

An increase in the apparent viscosity, $\mu_{\text {app }}$, from 0.00136 to $0.00393 \mathrm{~Pa} \mathrm{~s}$, results in an increase in bubble diameter by $10 \%$, provided no other parameter changes within the system. This increase in gas bubble diameter results in a subsequent decrease in specific surface area over which mass transfer can occur within the bioreactor, which can be expressed in Equation 8 (Paul, Atiemo-Obeng, \& Kresta, 2004). Therefore, any change in the bubble diameter will result in a corresponding change in the specific surface area.

$$
a=\frac{6 \phi_{\mathrm{g}}}{d_{\mathrm{b}}}
$$


where $a$ is the specific surface area $\left(\mathrm{m}^{-1}\right) ; \phi_{\mathrm{g}}$ is the gas holdup fraction.

Equation 8 also shows that the specific surface area is related to the gas holdup fraction, $\phi_{\mathrm{g}}$. The gas holdup fraction tends to be invariant with respect to the viscosity, being predominantly a function of the mixing intensity and the superficial gas velocity (Kawase \& Moo-Young, 1990). Therefore, the observed increase in viscosity resulted in a decrease in the specific surface area of $10 \%$.

The viscosity of a liquid also impacts the mixing within the process, resulting in an increase in the viscous dissipation of mixing energy. The mixing intensity within a reactor is generally assessed utilizing the Camp-Stein root mean square velocity gradient, $\bar{G}\left(\mathrm{~s}^{-1}\right.$; Crittenden, Trussell, Hand, Howe, \& Tchobanoglous, 2012). This measure of the velocity gradient is inversely proportional to the square root of the liquid dynamic viscosity, as detailed in Equation 9. As a consequence, increases in apparent viscosity result in decreases in the mixing energy within the system if the mixing power is the same. When looking at observed reactor performance, the change in apparent viscosity from 0.00136 to 0.00393 Pa s results in a decrease in $\bar{G}$ of approximately $41 \%$.

$$
\bar{G}=\left(\frac{P}{\mu_{\mathrm{app}} V_{\mathrm{R}}}\right)^{1 / 2} .
$$

As the mixing intensity is decreased, the scale of mixing increases, resulting in a reduction in the liquid-side mass transfer coefficient. Interpreting penetration theory through the lens of the theory of isotropic turbulence, it can be observed that the mass transfer coefficient is directly proportional to the mixing intensity, as indicated in Equation 10 (Kawase \& Moo-Young, 1990). Larger terminal eddies result in a thicker stagnant liquid film thickness at the air-liquid boundary, impeding mass transfer. An increase in the apparent viscosity from 0.00136 to $0.00393 \mathrm{~Pa}$ s results in a $21 \%$ decrease in the liquid film mass transfer coefficient, $k_{\mathrm{L}}$, assuming all other factors remaining equal.

$$
k_{\mathrm{L}}=\frac{2}{\sqrt{\pi}} D_{\mathrm{eff}}^{1 / 2}\left(\frac{\varepsilon}{v}\right)^{1 / 4}=\frac{2}{\sqrt{\pi}} D_{\mathrm{eff}}^{1 / 2} \bar{G}^{1 / 2} \propto \mu_{\mathrm{app}}^{-1 / 4},
$$

where $D_{\text {eff }}$ is the diffusivity of solute in a solvent $\left(\mathrm{m}^{2} / \mathrm{s}\right) ; \varepsilon$ is the average energy dissipation per unit mass $\left(\mathrm{J} \mathrm{kg}^{-1} \mathrm{~s}^{-1}\right)$; and $v$ is the kinematic viscosity $\left(\mathrm{m}^{2} / \mathrm{s}\right)$.

Viscosity may also affect the diffusivity of oxygen in water. Looking at established relationships for diffusivity of oxygen gas in water, they typically take the form indicated by Equation 11 (Blanch \& Clark, 1997) that was developed from the Stokes-Einstein equation.

$$
D_{\mathrm{O}_{2}, \mathrm{H}_{2} \mathrm{O}} \propto C \frac{T}{\mu_{\mathrm{o}}}
$$

where $D_{\mathrm{O} 2, \mathrm{H} 2 \mathrm{O}}$ is the diffusivity of oxygen in water $\left(\mathrm{m}^{2} / \mathrm{s}\right) ; C$ is a proportionality coefficient that is a function of solute spherical radius, molecular weight, etc.; $\mu_{\mathrm{o}}$ is the solvent viscosity, and $T$ is the liquid temperature $(\mathrm{K})$.
When considering the effects of the apparent viscosity of the mixed liquor samples utilized within the scope of this study, it is necessary to consider the fundamental mechanisms that drive variations in viscosity and their effects on the OTE. As was established when considering the $\mathrm{SV}_{30}$-Viscosity relationship, the mixed liquor is fundamentally a dispersion. The apparent viscosity is a result of the particles in suspension, and not an inherent property of the bulk solution. Even though the diffusivity can be impacted by the viscosity of the liquid, it is anticipated that for dispersions, the diffusivity will not change because the bulk solution viscosity does not change. As a consequence, oxygen will diffuse from the gas phase into the bulk liquid phase and into the interstitial water present within the hydrodynamic radius of the floc at the same rate as if the particles were not present, assuming that the particles do not block the gas-liquid interface or shuttle oxygen from the interface into the bulk solution.

To confirm that the changes in apparent viscosity were a property imposed by the particles and not an inherent property of the bulk solution, oxygen transfer testing was performed using clean water and effluents from all reactors. As demonstrated in Figure 5, there was no difference between the measured volumetric mass transfer rates obtained for all cases. Therefore, the change in apparent viscosity was induced by the sludge particles in suspension, and that diffusivity of oxygen within the bulk solution was unchanged. This testing also supports the assertion that no surface active agents were present which might affect OTE. Therefore, changes in the OTE for different reactors are a direct result of viscous dissipation of mixing energy, expansion of the liquid film thickness, and a reduction in the specific surface area over which mass transfer can occur. Interestingly, when the impacts of the apparent viscosity on the specific surface area of bubbles and the mass transfer coefficient are accounted for, it can be theoretically estimated that a $33 \%$ decrease in the OTE would be observed when the apparent

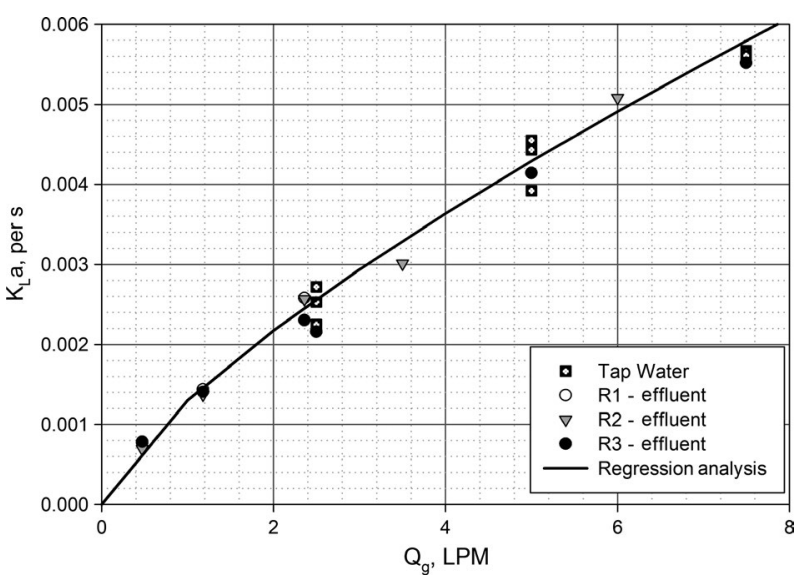

Figure 5. Volumetric mass transfer coefficient (KLa) versus the reactor airflow rate. The mixing intensity for all tests was maintained constant at $\bar{G}=150 \mathrm{~s}^{-1}$. The airflow was varied from 0.472 to $7.5 \mathrm{Lpm}$ for tap water (closed circles) and reactor effluent (open circles). A least squares regression analysis was performed, illustrating that no significant difference between the tap water and the reactor effluent exists. 
viscosity is increased from 0.00136 to $0.00393 \mathrm{~Pa}$, which is in reasonable agreement with the results observed in this study.

The implications of this finding are significant. The relationship between the high MLSS concentrations, elevated apparent viscosity, and OTE has previously been investigated. One common observation has been that a significant variability exists in the experimentally derived relationships between MLSS, apparent viscosity ( $<20 \mathrm{mPa}$ s), and OTE ( $\mathrm{Hu}, 2006$; Krampe \& Krauth, 2003). It is possible that these studies did not incorporate the impacts of filamentous microorganisms on the viscosity and OTE measurement. For example, Gil et al. (2011) observed a generally linear relationship $\left(R^{2}=0.65\right)$ between MLSS and the apparent viscosity of mixed liquor culture in an MBR. Subsequent testing performed for bulking sludge demonstrated a very strong linear MLSS-apparent viscosity response $\left(R^{2}=0.96\right.$; Gil et al., 2011). The presence of filamentous organisms tends to be a continuum, ranging from no filaments to an excessive abundance of filaments (Jenkins, Richard, \& Diagger, 1986). A minimal concentration of filaments is necessary to form the strong backbone of the microbial floc. It is likely that the results reported by Gil et al. for the normal mixed liquor had varying concentrations of filamentous organisms, which influenced viscosity and subsequently the OTE. The bulking sludge had a high density of filamentous organisms, significantly reducing the variability in the recorded data. As we demonstrated, the apparent viscosity and OTE are strongly related, meaning any variance in the MLSS-viscosity data will also result in variance in the OTE. Future reports of OTE in the activated sludge process must account for microbial morphology to provide a complete assessment of actual system performance and to allow for direct and meaningful comparisons between reports.

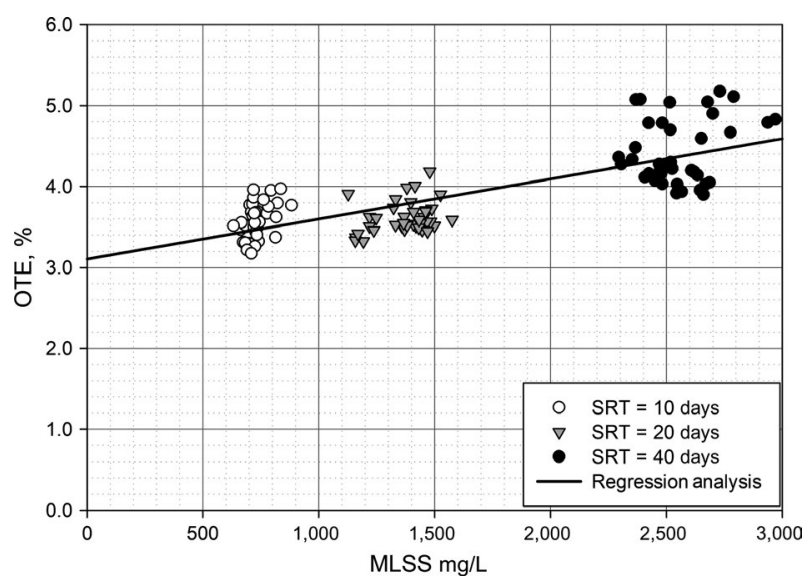

Figure 6. Oxygen transfer efficiency (OTE) versus mixed liquor suspended solids (MLSS) concentration. A linear relationship appears to exist between the MLSS and the OTE $\left(R^{2}=0.62\right)$, demonstrating an increase in the OTE with increasing MLSS. It must be noted, that here the MLSS concentrations reported are a corollary to the reactor SRT, with the 40-day SRT reactor exhibiting significantly improved OTE as compared to the 10-day SRT reactor. Data reported since the commencement of viscosity testing (Day 400).

\section{Solids retention time}

Figure 6 demonstrates that there is an increasing trend in the OTE with increasing SRT. Here, the MLSS is a corollary for the reactor SRT, which was controlled via the wasting rate. The linear trend was confirmed through regression analysis $\left(R^{2}=0.62\right)$. The OTE increased by approximately $26 \%$ as the MLSS was increased from 740 to $2,580 \mathrm{mg} / \mathrm{L}$. This observed increase in OTE was significant, resulting in a reduction in the air delivery rate to the 40-day SRT reactor, compared with the 10-day reactor.

It must be stressed that influent wastewater composition was strictly controlled, minimizing the potential for the presence of surface active agents within the reactor. Furthermore, oxygen transfer testing revealed that the volumetric mass transfer coefficients for all reactor effluents were similar to those observed for clean water (see Figure 5). These observations demonstrate that the property of the bulk solution was not responsible for reactor performance; sludge morphological parameters were.

The finding that a higher SRT-activated sludge process promotes oxygen transfer supports the earlier observations (Leu, Chan, \& Stenstrom, 2012; Rosso, Iranpour, \& Stenstrom, 2005; Rosso, Stenstrom, \& Larson, 2008). Furthermore, this finding highlights one of the many performance-related benefits reaped by long SRT processes. Increased oxygen demand has long been a concern for long SRT operation due to the enhanced role of endogenous respiration. Yet the aforementioned results demonstrate that long SRT should not be a concern. As was demonstrated with this study, better OTE of the 40-days SRT reactor resulted in 7\% less air delivery than for the 10-day SRT reactor. Less air delivery equates to a reduction in energy consumption associated with the process. Long SRT reactors are also known to have improved process stability as a result of increased active biomass concentrations, providing capacity to handle shock loads. As was demonstrated in this study, the long SRT reactor had improved settleability as compared to its shorter SRT counterparts. The improved settleability promotes an enhanced solids flux, allowing the process to be operated at a higher solids concentration without detriment to the solids separation process. Long SRT operation also significantly reduces biomass production, allowing a reduction in sludge pumping, thickening, stabilization and dewater process sizes, saving energy, site space, and capital. The waste sludge that is produced tends to have better filterability, stabilization, and dewatering characteristics than observed for short SRT processes, reducing the effort necessary to prepare the sludge for its final disposition. Finally, the long SRT-activated sludge process provides the capability for the better degradation of recalcitrant waste compounds such as pharmaceuticals and personal care products (Leu et al., 2012).

\section{Statistical analysis}

A Best Subset Regression Analysis was performed for all measured data to determine linear combinations of factors having the most significant impact on the OTE. The analysis was performed utilizing the coefficient of determination as means of identifying parameters of importance. Based on the analysis, 
viscosity was a statistically significant $(p<0.05)$ descriptor of the OTE, having a low variance inflation factor $(\mathrm{VIF}<5)$ and a high coefficient of determination $\left(R^{2}=0.720\right)$. The coefficient for viscosity was negative, indicating a reduction in OTE with an increase in viscosity.

\section{Conclusions}

Oxygen transfer performance of three complete-mix activated sludge bioreactors operated under SRTs of 10, 20, and 40 days were investigated. Results indicated that the filament density in the activated sludge affects not only the sludge settleability, but also the oxygen transfer performance at the air-liquid boundary. Increasing $\mathrm{SV}_{30}$ from 20 to 100 decreases OTE by $28 \%$. This is because a greater filament density results in a greater sludge volume concentration in the mixing liquor therefore a greater apparent viscosity. A greater viscosity results in a greater air bubble size and thicker liquid film thickness at the air-liquid boundary, both of which reduce oxygen transfer performance. Results also indicated that the 40-day SRT reactor has the lowest filament density therefore the highest OTE compared to the 10- and 20-day SRT reactors. Increasing SRT from 10 day to 40 day increases the OTE by $26 \%$, and this OTE increase results in a net reduction in air supply rate by $7 \%$. Operating an activated sludge process at a very long SRT may reduce aeration energy use, in addition to other benefits such as reduced sludge production and improved removal of recalcitrant contaminants.

\section{ACKNOWLEDGMENTS}

Rheological testing of activated sludge was made possible via Center for Infrastructure Engineering Studies, Missouri University of Science and Technology. Feedback provided by Dr. C.P. Huang at the University of Delaware regarding viscosity of dispersions is greatly appreciated.

\section{CONFLICT OF INTEREST}

The authors declare that no conflicts of interest exist relating to commercial, financial, or personal relationships and the performance of this research.

\section{REFERENCES}

APHA, AWWA, and WEF (2009). Standard methods for the examination of water and wastewater (20th ed.). Washington, DC: American Public Health Association.

ASCE (1997). ASCE/EWRI-18-96: Standard guidelines for in-process oxygen transfer testing. New York, NY: American Society of Civil Engineers.

ASCE (2007). ASCE/EWRI 2-06: Measurement of oxygen transfer in clean water. Reston, VA: American Society of Civil Engineers.

Bhavaraju, T. W. F., \& Russel, H. W. B. (1978). The design of gas sparged devices for viscous liquid systems. AIChE Journal, 24(3), 454-466. https://doi.org/10.1002/ (ISSN)1547-5905

Blanch, H. W., \& Clark, D. S. (1997). Biochemical engineering. Boca Raton, FL: CRC Press. Crittenden, J. C., Trussell, R. R., Hand, D. W., Howe, K. J., \& Tchobanoglous, G. (Eds.) (2012). MWH's Water treatment: Principles and design. Hoboken, NJ: John Wiley \& Sons Inc.
Gabb, D. M. D., Ekama, G. A., Jenkins, D., \& Marais, G. V. R. (1989). Incidence of Sphaerotilus natans in laboratory scale activated sludge systems. Water Science and Technology, 21(4/5), 29-41. https://doi.org/10.2166/wst.1989.0208

Gil, J. A., Krzeminski, P., van Lier, J. B., van der Graaf, J. H. J. M., Wijffels, T., \& Prats, D. (2011). Analysis of the filterability in industrial MBRs. Influence of activated sludge parameters and constituents on filterability. Journal of Membrane Science, 385-386, 96-109. https://doi.org/10.1016/j.memsci.2011.09.030

Grady, J., Leslie, C. P., Daigger, G. T., \& Lim, H. C. (1999). Biological wastewater treatment, second edition, revised and expanded. New York, NY: Marcel Dekker Inc.

Hiemenz, P. C., \& Rajagopalan, R. (1997). Principles of colloid and surface chemistry. New York, NY: Marcel Dekker Inc. https://doi.org/10.1201/9781315274287

$\mathrm{Hu}, \mathrm{J}$. (2006). Evaluation of parameters influencing oxygen transfer effeciency in a membrane bio reactor. Honolulu, HI: University of Hawai'i.

Jenkins, D., Richard, M. G., \& Diagger, G. T. (1986). Manual on the causes and control of activated sludge bulking and foaming. Pretoria, South Africa: Water Research Commission.

Ju, F., \& Zhang, T. (2014). Bacterial assembly and temporal dynamics in activated sludge of a full-scale municipal wastewater treatment plant. The ISME Journal, 9, 683.

Kawase, Y., \& Moo-Young, M. (1990). Mathematical models for design of bioreactors: Applications of Kolmogoroff's theory of isotropic turbulence. The Chemical Engineering Journal, 43, B19-B41. https://doi.org/10.1016/0300-9467(90)80048-H

Krampe, J., \& Krauth, K. (2003). Oxygen transfer into activated sludge with high MLSS concentrations. Water Science and Technology, 47(11), 297-303. https://doi. org/10.2166/wst.2003.0618

Leu, S.-Y., Chan, L., \& Stenstrom, M. K. (2012). Toward long solids retention time of activated sludge processes: Benefits in energy saving, effluent quality, and stability. Water Environment Research, 84(1), 42-53. https://doi.org/10.2175/1061430 11 X12989211841052

Liu, G., \& Wang, J. (2013). Long-term low DO enriches and shifts nitrifier community in activated sludge. Environmental Science \& Technology, 47(10), 5109-5117. https:// doi.org/10.1021/es304647y

Liu, G., \& Wang, J. (2015). Modeling effects of DO and SRT on activated sludge decay and production. Water Research, 80, 169-178. https://doi.org/10.1016/j. watres.2015.04.042

Liu, G., Wang, J., \& Campbell, K. (2018). Formation of filamentous microorganisms impedes oxygen transfer and decreases aeration efficiency for wastewater treatment. Journal of Cleaner Production, 189, 502-509. https://doi.org/10.1016/j.jclepro.2018.04.125

Macosko, C. (1994). Rheology: Principles, measurements and applications. New York, NY: Wiley-VCH Inc.

Martins, A. M. P., Pagilla, K., Heijnen, J. J., \& van Loosdrecht, M. C. M. (2004). Filamentous bulking sludge-A critical review. Water Research, 38(4), 793-817. https://doi. org/10.1016/j.watres.2003.11.005

Meng, F., Zhang, H., Yang, F., Li, Y., Xiao, J., \& Zhang, X. (2006). Effect of filamentous bacteria on membrane fouling in submerged membrane bioreactor. Journal of Membrane Science, 272(1), 161-168. https://doi.org/10.1016/j.memsci.2005.07.041

Palm, J. C., Jenkins, D., \& Parker, D. S. (1980). Relationship between organic loading, dissolved oxygen concentration and sludge settleability in the completely-mixed activated sludge process. Journal - Water Pollution Control Federation, 52(10), $2484-2506$.

Paul, E. L., Atiemo-Obeng, V. A., \& Kresta, S. M. (2004). Handbook of industrial mixing: Science \& practice. Hoboken, NJ: Wiley-Interscience.

Rosso, D., Iranpour, R., \& Stenstrom, M. K. (2005). Fifteen years of offgas transfer efficiency measurements on fine-pore aerators: Key role of sludge age and normalized air flux. Water Environment Research, 77(3), 266-273. https://doi. org/10.2175/106143005X41843

Rosso, D., Stenstrom, M. K., \& Larson, L. E. (2008). Aeration of large-scale municipal wastewater treatment plants: State of the art. Water Science \& Technology, 57(7), 973-978. https://doi.org/10.2166/wst.2008.218

Sarioglu, M., Insel, G., Artan, N., \& Orhon, D. (2009). Model evaluation of simultaneous nitrification and denitrification in a membrane bioreactor operated without an anoxic reactor. Journal of Membrane Science, 337(1-2), 17-27. https://doi.org/10.1016/j. memsci.2009.03.015

Sezgin, M. (1982). Variation of sludge volume index with activated sludge characteristics. Water Research, 16, 83-88. https://doi.org/10.1016/0043-1354(82)90056-2

Sezgin, M., Jenkins, D., \& Parker, D. S. (1978). A unified theory of filamentous actived sludge bulking. Journal Water Polution Control Federation, 50(2), 362-381.

US Department of Energy (2014). The water-energy Nexus: Challenges and opportunities (p. 240). Washington, DC: US Department of Energy.

Valentín-Vargas, A., Toro-Labrador, G., \& Massol-Deyá, A. A. (2012). Bacterial community dynamics in full-scale activated sludge bioreactors: Operational and ecological factors driving community assembly and performance. PLoS ONE, 7(8), e42524. https://doi.org/10.1371/journal.pone.0042524

Wagner, M., Cornel, P., \& Krause, S. (2002). Efficiency of different aeration systems in full scale membrane bioreactors. In Proceedings, Water Environment Federation (pp. 434-443). Alexandria, VA.

WEF and ASCE (2010). Design of municipal wastewater treatment plants: WEF manual of practice no. 8, ASCE manuals and reports on engineering practice no. 76 (5th ed.). Alexandria, VA; Reston, VA; New York, NY: WEF Press; American Society of Civil Engineers, Environmental and Water Resources Institute; McGraw-Hill. 